\title{
Meta-Analysis: Relationship Knowledge, Attitude and Information Media of Reproductive Health Problems on Adolescent Sexual Behavior
}

\author{
Dyah Komala Rosyita * \\ Faculty of Medicine, Lambung Mangkurat \\ University, Banjarbaru, Indonesia \\ Meitria Syahadatina Noor \\ Faculty of Medicine, Lambung Mangkurat \\ University, Banjarbaru, Indonesia
}

\author{
Roselina Panghiyangani \\ Faculty of Medicine, Lambung Mangkurat \\ University, Banjarmasin, Indonesia \\ Husaini \\ Faculty of Medicine, Lambung Mangkurat \\ University, Banjarbaru, Indonesia
}

\author{
Lenie Marlinae \\ Faculty of Medicine, Lambung Mangkurat \\ University, Banjarbaru, Indonesia
}

\begin{abstract}
Adolescence is one of the two periods of an individual's life span, where there are very large physical changes, namely the maturation of reproductive organs and functions. This behavior can have a detrimental impact on adolescents themselves, such as contracting STIs and HIV and AIDS, pregnancy out of wedlock, abortion, physical, social, and psychological disorders. The results of the WHO survey in 2015 averaged 44.1 deaths among female adolescents aged 15-19 years due to sexual behavior and reproductive health problems. Meanwhile, the average death rate for adolescents (15-19 years) due to childbirth in Indonesia is 47 people. This study analyzes the relationship between knowledge, attitudes, and access to information on reproductive health issues with adolescent sexual behavior. The method used is Meta-analysis by searching the Google Scholar database. After going through the identification and selection stages, 13 articles were included in the meta-analysis. Pooled Odds Ratio (POR) was calculated using a fixed-effect model for data analysis according to the heterogeneity test using Review Manager 5.4.1. Based on the review, the POR value for knowledge is 2.07 (95\% CI 1.29-3.33), attitude 2.17 (95\% CI 1.04-4.56), and Information Media 3.95 (95\% CI 1.81-8.861), so that it can be concluded from the three variables studied, the information media variable has a stronger effect than the knowledge and attitude variables towards adolescent sexual behavior.
\end{abstract}

Keywords: Knowledge, attitude, information media, reproductive health, adolescent sexual behavior

Received: 12 December 2019; Accepted: 30 January 2020; Published: 28 March 2020

\section{INTRODUCTION}

Adolescent sexual behavior is all behavior that is driven by sexual desire, both with the opposite sex and with the same sex [1]. This behavior can be done before marriage or during courtship, and it is called pre-marital sexual behavior. Sexual behavior is behavior based on sexual urges or activities to get pleasure from the sexual organs through various behaviors. The forms of behavior vary from feelings of attraction to the behavior of dating, making out, and having sex [2].

Concerning these changes, adolescents also begin to

\footnotetext{
${ }^{*}$ Correspondence concerning this article should be addressed to Dyah Komala Rosyita, Faculty of Medicine, Lambung Mangkurat University, Banjarbaru, Indonesia. E-mail: dyahkomala39@gmail.com

(C) 2020 The Author(s). Published by KKG Publications. This is an Open Access article distributed under a Creative Commons AttributionNonCommercial-NoDerivatives 4.0 International License.
} 
feel a sexual urge and show interest in the opposite sex. As a result, teenagers start to experiment in terms of sexuality. Deviant sexual behavior that adolescents often carry out includes masturbation, petting, and sexual intercourse $[3,4]$.

Pre-marital sex performed on adolescents can cause negative things, one of which is unwanted pregnancies and the sexually transmitted disease HIV/AIDS. Unwanted pregnancies lead to abortions and teenage marriages, both of which will impact their future, the fetus they are carrying, and their family.

Teenage pregnancy has a negative impact on the health of adolescents and their babies, and it can also have social and economic impacts. Pregnancy at a young age or adolescence includes the risk of premature birth, Low Birth Weight (LBW), childbirth bleeding, increasing maternal and infant mortality. Teenage pregnancies are also associated with unwanted pregnancies and unsafe abortions.

In the current era of globalization, teenagers are very sophisticated in using the internet, as known for social media users it reaches 2.2 billion with users reaching 3.7 billion from the 2016 APJII survey results about internet users in Indonesia, 8.3 million students are using the internet, based on the 10-24-year-old age group, $75.5 \%$ are active internet users and access to social media is the largest content, namely $97.4 \%$ or 129.2 million people [5]. Where based on the data obtained, the reason why adolescents aged 15-24 years had sexual intercourse for the first time was that as many as (54\% of women) and ( $46 \%$ of men) had sexual relations with the reason of loving each other, then another reason put forward by men was because of curiosity (34\%), and women because they were forced by their partners (16\%), then other reasons were because it just happened (16\% women) and (15\% men).

In Indonesia, teenagers, on average, date for the first time in the age range of 15-17 years; at this age, teenagers are prone to risky sexual behavior that will lead to premarital sexual activity. As many as $85 \%$ of adolescents hold hands while dating, $47 \%$ hug, $48.1 \%$ have kissed lips, $6.2 \%$ have stimulated their partner's genitals, and $19.1 \%$ have had pre-marital sexual activities.

South Kalimantan Province is one of the areas in Indonesia with a high risk of transmitting HIV/AIDS, which is indicated by the continued increase in the spread of the deadly disease in 13 local regencies and cities. Meanwhile, the highest record for PLWHA 2018 in terms of regions according to the South Kalimantan AIDS Commission (KPA) data, the first position was occupied by Banjarmasin City with 647 people, followed by Tanah
Bumbu 294 people, Banjabaru in third 236 people and Banjar Regency with 117 people. In terms of employment criteria, 5 schoolchildren and students live with HIV/AIDS in the Banjarbaru area.

Data from the 2019 South Kalimantan Balitbangda stated that 22.15 percent of Banua's women aged less than 16 years chose to get married. Meanwhile, at 17 to 18 years, 23.32 percent are already sitting on the aisle. If viewed by district/city, early marriages under 16 were mostly in Balangan last year. In this area, 33.27 percent of women under the age of 16 are married. At the same time, the lowest was in Banjarbaru, with a percentage of 13.34 percent. The percentage of early marriages collected are those reported. Perhaps there are many more that have not been recorded. If viewed since 2017, the percentage of young women who marry early has decreased, although the decline is very small. The percentage of women under the age of 16 who were ever married based on age at first marriage in 2017 reached 22.33 percent. Then, in 2018 it rose to 22.77 percent, and in 2019 , it fell to 22.15 percent [6].

According to the results of research conducted by [7], The results of the chi-square statistical test at $=0.05$ obtained a $p$-value of $0.000(p<0.05)$, which states that there is a significant relationship between knowledge of reproductive health knowledge and adolescent sexual behavior. This is contrary to the research conducted by [8]. From the statistical tests carried out using the chi-square test, it was obtained that $p=0.214>\alpha=0.05$, which stated that there was no significant relationship between knowledge of reproductive health and adolescent sexual behavior.

Based on the results of the chi-square statistical test, the results obtained with a value of $p=0.002$, which means the value of $=0.05(p<0.05)$, it can be concluded that there is a relationship between attitudes and sexual behavior in adolescents. And the results of [9] research with statistical test results using the ChiSquare formula obtained $p=0.007(p<0.05)$, which means a relationship between information media and sexual behavior in adolescents.

Based on the description above, it can be seen that there are differences in the results between one study and another, where many studies have been conducted to determine the risk factors associated with adolescent sexual behavior. In general, the research results show a relationship between knowledge, attitudes, and access to information on reproductive health issues on adolescent sexual behavior. However, some find that there is no relationship between these variables.

Therefore, it is considered important to conduct a 
meta-analysis study by combining several research results regarding the relationship between knowledge, attitudes, and access to information on reproductive health issues on adolescent sexual behavior to determine the relationship between these variables. Research with meta-analysis is a technique used to summarize the findings of two or more studies to combine, review, and summarize previous research. In addition, by using meta-analysis, various questions can be investigated based on data that has been found from the results of previous studies that have been published, and one of the requirements needed in conducting a meta-analysis is an assessment of the results of similar research. One of the advantages of meta-analysis is that new studies with many subjects can draw more definitive conclusions [10].

\section{METHOD}

\section{A. Research Design}

This type of research is a meta-analysis study where the researcher uses several similar research articles to combine so that new data is obtained and quantitative testing is carried out.

In this meta-analysis, an observational study was conducted to estimate the effect size, namely the strength of the relationship or the magnitude of the difference between variables [11].

\section{B. Research Stages}

The stages in this research are as follows:

1) Data quality analysis:

2) Identify research questions: This stage is the first step, which is a question to be used to conduct a review. The SPIDER method was chosen because the articles used as references did not involve intervention.

3) Research protocol: The research protocol is a detailed plan that includes the scope of the study, procedures, criteria for assessing quality (inclusion and exclusion criteria), the scale of the research to be carried out. The protocol of this study uses the concept of Preferred Reporting Items for Systematic Reviews and Meta-Analysis (PRISMA).

4) Search strategy: The search strategy was carried out referring to the research protocol made and determining the location or source of the database for data retrieval.
5) Data extraction: Data extraction was carried out after the research protocol process had been carried out using the PRISMA method. Data extraction was carried out manually by making a list of journals containing: serial number, year of the journal, the title of the journal, name of the researcher, name of the journal, data source, purpose, results, technical data analysis, sample, variables studied, and research results (OR/ AOR, $p$-value, and CI).

\section{Population, Sample, and Sampling Technique}

1) Population: The population is the whole object of research or also called the universe

2) Sample: The sample consists of part of the population that can be used as research subjects through sampling by considering the inclusion criteria. As a sample in research, 10 articles were studied.

3) Sampling technique: The sampling technique is used in sampling to obtain an appropriate sample of the entire research subject.

4) Research variables: The research variables show the variables studied, consisting of 1. Independent Variable The independent variables in this study were knowledge, attitudes, and access to information on reproductive health issues. 2. Dependent variable

The dependent variable in this study is adolescent sexual behavior. After conducting the study selection, all relevant research will be systematically reviewed, and several variables will be taken (abstraction). The amalgamation of results from various studies is the most decisive part of the meta-analysis. Data analysis can use the fixedeffect model or random-effect model.

\section{RESULTS AND DISCUSSION}

\section{A. Meta-Analysis of the Relationship Between Knowl- edge and Adolescent Sexual Behavior}

The number of research articles combined to analyze the relationship between knowledge and adolescent sexual behavior was 11 articles, 10 (ten) articles stating that they were related, and 1 (one) which stated that they were not related. The eleven research articles are research with a cross-sectional design. The following are the results of the meta-analysis of the relationship between knowledge and adolescent sexual behavior: 


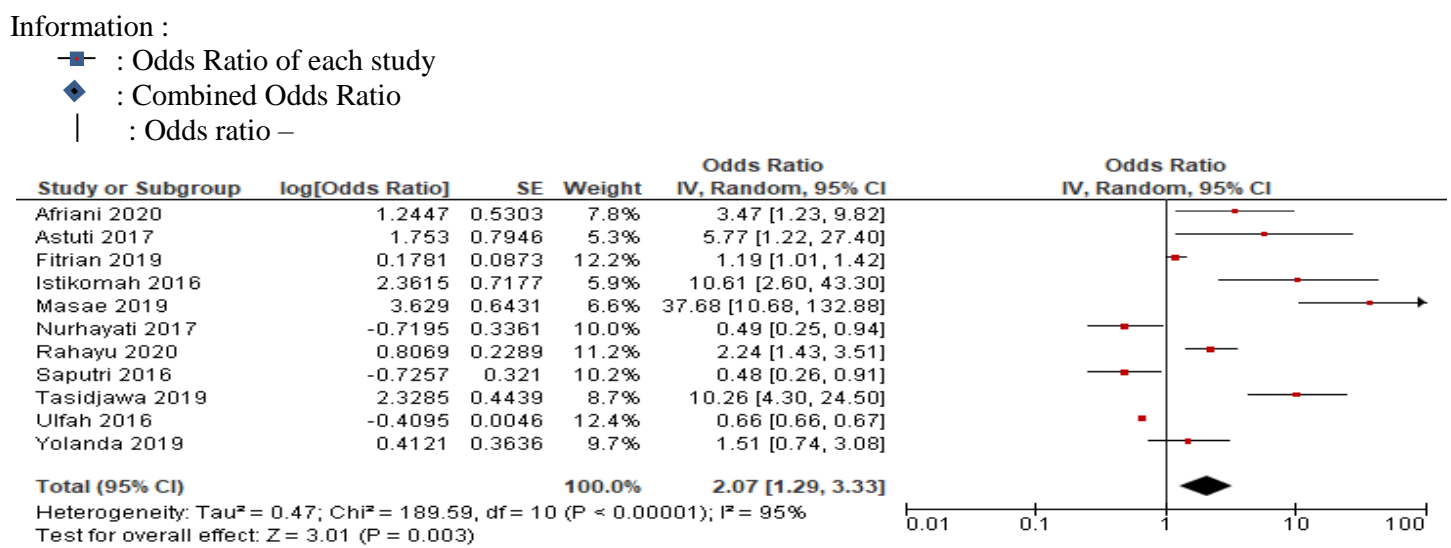

Fig. 1. Forest plot research study of the relationship between knowledge and adolescent sexual behavior

In Fig. 1, the forest plot results from the synthesis in the meta-analysis that describes the conclusions drawn from several similar studies combined with the effect size value of each study and the combined effect size value. In the forest plot, there is a small black box ( $\mathbf{\square})$ this is the estimated effect in each study and (-) the horizontal line in the small box is the $95 \%$ CI value, the narrow horizontal line of the CI value indicates good precision and is always more informative than the CI wide, if the sample is small, the horizontal line will be wide. Then in the form of diamond $(\diamond)$ which is the effect size value of the combined study with a certain confidence interval.

Fig. 1 shows the results of data analysis from 11 articles that examine the relationship of knowledge with adolescent sexual behavior analyzed using the Random Effect Model to measure the magnitude of the combined effect of the data in the data input model in the Revman 5.4.1 application. The heterogeneity test results showed that the study variation was heterogeneous with a $p$-value of $<0.05$, namely $p=0.0003$, and the value of variation between studies (I2) was 95\% means very high heterogeneity.

The results of data analysis on the forest plot show that there is a relationship between knowledge and adolescent sexual behavior, with a $p$-value of $<0.005$, namely $p$ $=0.003$, and a pooled odds ratio of 2.07 with a $95 \%$ confidence interval (1.29-3.33), so it can be concluded that adolescents who have a low level of knowledge tend to be at risk of 2.07 times having pre-marital sexual behavior in adolescents.

\section{B. Meta-Analysis of the Relationship Between Attitudes and Adolescent Sexual Behavior}

The number of research articles combined to analyze the relationship between attitudes and adolescent sexual behavior was 7 articles. Five articles stated that they are related, and two state that they are not related. The seven research articles are research with a cross-sectional design. The following are the results of the meta-analysis of the relationship between attitudes and adolescent sexual behavior:

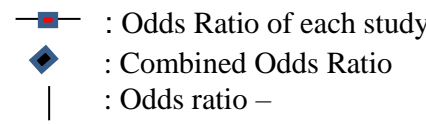

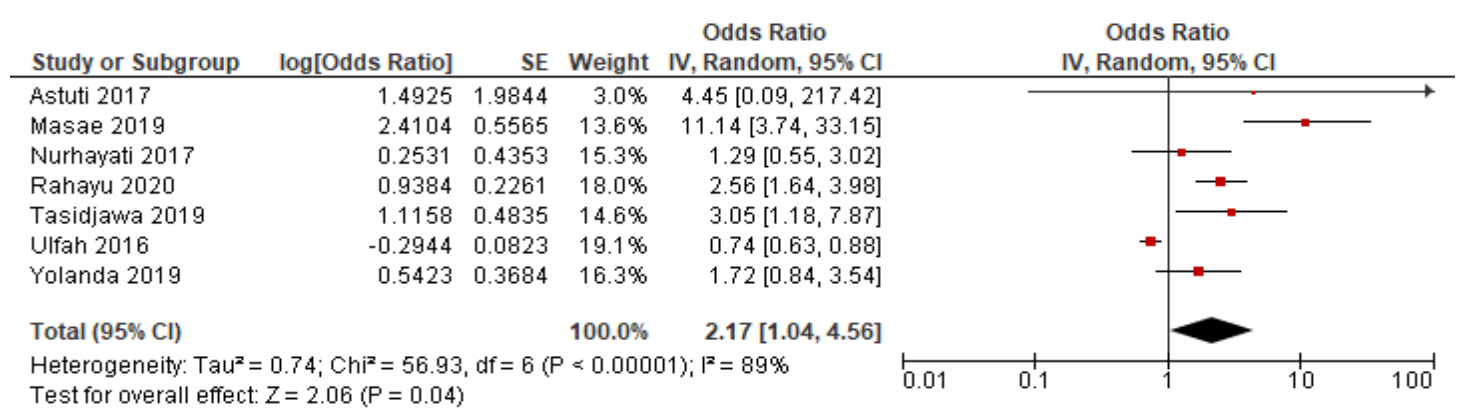

Fig. 2. Forest plot research study of the relationship between attitudes and adolescent sexual behavior 
In Fig. 2 forest plot is the result of synthesis in the meta-analysis that describes the conclusions drawn from several similar studies, which are combined with the effect size value of each study and the combined effect size value. In the forest plot, there is a small black box ( $\boldsymbol{\square}$ ) this is the estimated effect in each study and (-) the horizontal line in the small box is the $95 \%$ CI value, the narrow horizontal line of the CI value indicates good precision and is always more informative than the $\mathrm{CI}$ wide, if the sample is small, the horizontal line will be wide. Then in the form of diamond ( $)$ which is the value of the combined effect size of the study with a certain confidence interval.

Fig. 2 shows the results of data analysis from 7 articles that examine the relationship between attitudes and adolescent sexual behavior analyzed using the Random Effect Model to measure the magnitude of the combined effect of the data input model in the Revman 5.4.1 application. The heterogeneity test results showed that the study variation was heterogeneous, with a value of $p=$ 0.04 , meaning that heterogeneity was very high because $p<0.05$ and the value of variation between studies (I2) was $89 \%$.

The results of data analysis on the forest plot show that there is a relationship between attitudes and adolescent sexual behavior with a $p$-value of $<0.005$, namely $p$ $=0.004$ and a pooled odds ratio of 2.17 with a confidence interval of $89 \%$ (1.04-4.56), so it can be concluded that adolescents who Having a negative attitude tend to be 2.17 times more likely to engage in pre-marital sexual behavior than adolescents who have a positive attitude.

\section{Meta-Analysis of the Relationship Between Informa- tion Media and Adolescent Sexual Behavior}

The number of research articles combined to analyze the relationship between information media and adolescent sexual behavior was eight. Six articles stated that they are related, and two states that they are not related. The eight research articles are research with a cross-sectional design. The following are the results of a meta-analysis of the relationship between information media and adolescent sexual behavior.

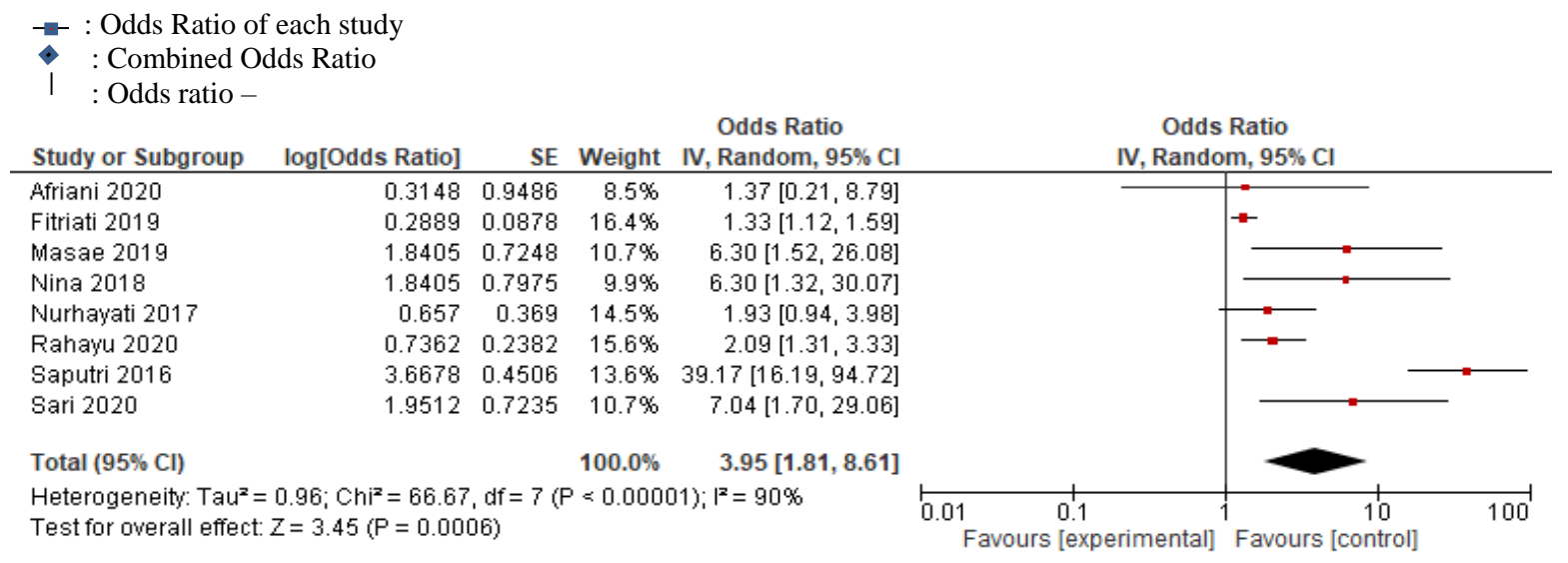

Fig. 3. Forest Plot research study of the relationship between information media and adolescent sexual behavior.

In Fig. 3, the forest plot results from the synthesis in the meta-analysis that describes the conclusions drawn from several similar studies combined with the effect size value of each study and the combined effect size value. In the forest plot, there is a small black box ( $\square$ ) this is the estimated effect in each study and (-) the horizontal line in the small box is the $95 \%$ CI value, the narrow horizontal line of the CI value indicates good precision and is always more informative than the CI wide, if the sample is small, the horizontal line will be wide. Then in the form of diamond $(\checkmark)$ which is the value of the combined effect size of the study with a certain confidence interval.

Fig. 3 shows the results of data analysis from 8 articles that examine the relationship of information media with adolescent sexual behavior was analyzed using the Random Effect Model to measure the magnitude of the combined effect of the data in the data input model in the Revman 5.4.1 application. The heterogeneity test results showed that the research variation was heterogeneous with a $p$-value of $<0.05$, namely $p=0.0006$, and the value of variation between studies (I2) was $90 \%$. It means very high heterogeneity.

The results of data analysis on the forest plot show that there is a relationship between knowledge and adolescent sexual behavior, with a $p$-value of $<0.005$, namely $p$ $=0.006$ and a pooled odds ratio of 3.95 with a confidence interval of $90 \%$ (1.81-8.861), so it can be concluded that adolescents who are exposed to information media tend to 
be at risk of 3.95 times doing pre-marital sexual behavior.

\section{DISCUSSION}

A. Meta-Analysis of the Relationship between Knowledge and Adolescent Sexual Behavior

\section{B. Research variation and heterogeneity:}

The test results show high heterogeneity, so that using random-effect models in measuring the combined effect of the data in the data input model in the Revman 5.4.1 application. The results obtained are POR $=2.07$ with a $95 \%$ confidence interval (1.29-3.330. The combined effect also produces a $Z$-value of 3.01 and $p$-value $<0.0001$. Statistically, the combined effect is significant if the $p$-value is $<0.05$, and this means that the null hypothesis is rejected. In other words, there is a relationship between knowledge and sexual behavior of adolescents having low knowledge increases the risk of 2.07 times having low knowledge pre-marital sexual behavior in adolescents. Eleven studies analyzed using Revman 5.4.1 can be seen that there is a very high heterogeneity bias value based on the funnel plot.

1) Analysis of research results: The results of data analysis from this study indicate a relationship between knowledge and adolescent sexual behavior. This is evidenced by the overall effect $p$ test value of less than $0.05, p=0.003$. The pooled odds ratio value is 2.07 with a $95 \%$ confidence interval that is $1.29-3.33$, so that it can be concluded that adolescents who have a low level of knowledge tend to be at risk of 2.07 times having pre-marital sexual behavior in adolescents. The mean effect size is 0.4064 , which means that knowledge has a moderate effect on adolescent sexual behavior.

Based on the effect size value results, the largest value in the group of related articles was 0.417 , which showed a moderate effect trend, so the researcher concluded that knowledge was related to adolescent sexual behavior with a moderate effect tendency. The average value of the largest effect size is 0.417 from the analysis of the group of articles that state a relationship between knowledge and sexual behavior of adolescents, while the average effect size of the group of articles that state that there is no relationship is 0.4064 . So it can be concluded that knowledge has a relationship with adolescent sexual behavior and has a moderate effect on anemia in adolescent girls.

2) Comparison of meta-analysis results: From the results of article synthesis, there are ten articles that state that there is a relationship between knowledge and adolescent sexual behavior, namely the research of $[12,13,14,3,15,16,17,18]$. In [3], adolescent sexual behavior based on knowledge shows that adolescents who have low knowledge tend to be 3.6 times at risk of having pre-marital sexual behavior in adolescents $(\mathrm{OR}=3,629$ (95\% CI 10,682-132, 886)

In addition to seeing the magnitude of the combined effect of the 11 journals, the funnel plot images in this study represent the presence of bias or not. The funnel plot shows publication bias. According to [19], the plot will form a symmetrical distribution if there is no bias. If there is a bias, the funnel plot will appear asymmetrical with gaps on the right and left sides of the graph. The funnel plot in this study shows a publication bias indicated by the plot's asymmetric distribution (dots) bounded by vertical lines. This bias may be because smaller studies did not show a statistically significant effect and were not published, resulting in a publication bias. Funnel plot asymmetry is often attributed to publication bias without consideration of other possibilities.

\section{Meta-Analysis of the Relationship between Attitudes and Adolescent Sexual Behavior}

1) Research variation and heterogeneity: The results of the analysis show different values of variance and weights. The theory expressed by [20] is that the weight in a study is directly proportional to the number of research subjects (research samples). Research with 430 subjects will have a greater weight than research with 21 subjects. In addition to the number of subjects, the weight is also influenced by variations in the data. The weights are inversely proportional to the variation of the data. Research with more varied data will have a greater weight than research with smaller variations.

The test results show high heterogeneity, so that using random-effect models in measuring the combined effect of the data in the data input model in the Revman 5.4.1 application. The results obtained are POR $=2.17$ with an $89 \%$ confidence interval (1.04-4.56). The combined effect also produces a $Z$ value of 2.06 and a $p$-value of $<$ 0.0001 . Statistically, the combined effect is significant if the $p$-value $<0.05$, and this means that the null hypothesis is rejected. In other words, there is a relationship between attitudes and adolescent sexual behavior; having a negative attitude increases the risk of 2.17 times pre-marital sexual behavior in adolescents. Seven studies analyzed using Revman 5.4.1 show a very high level of heterogeneity bias based on the funnel plot.

2) Analysis of research results: This study is the first meta-analysis study that analyzes the relationship between knowledge and adolescent sexual behavior. Many studies have analyzed the knowledge associated with adolescent sexual behavior. For this reason, statistical anal- 
ysis was carried out using meta-analysis to prove the quality of each study so that new data that was quantitative in nature and more accurate conclusions could be drawn.

Based on the test results through the Revman 5.4.1 application, the POR value $=2.17$ with an $89 \%$ confidence interval of 1.04-4.56. The combined effect also resulted in a $Z$ value of 2.06 and a $p$-value of $<0.04$. Statistically, the combined effect was significant if the $p$-value $<0.05$. This means that it can be concluded that the null hypothesis is rejected; in other words, there is a relationship between attitudes and sexual behavior; it is concluded that adolescents who have low levels of knowledge tend to be 2.17 times at risk of having pre-marital sexual behavior in adolescents.

3) Comparison of meta-analysis results: In the results of the study, seven related articles stated that there was a relationship between information media and adolescent sexual behavior indicated by a $p$-value $<0.05$, i.e., 0.04 Research which stated that there was a relationship between 5 articles with a sample of 917 and 2 articles that had no relationship with a total sample of 291 .

Five studies state a relationship between information media and adolescent sexual behavior, namely the [12] study with an OR value of 3.05. [3] with an OR value of 111.11 , [15] with an OR value of 4.48, with an OR value of 2.55, and [18] with an OR value of 0.74 . That shows a relationship between attitudes and adolescent sexual behavior.

According to the researcher's assessment, adolescents who have negative attitudes about reproductive health will impact pre-marital sexual acts. Knowing the importance of adolescent reproductive health knowledge will change the negative attitudes of adolescents towards sexual behavior

4) Meta-Analysis of the Relationship between Information Media and Adolescent Sexual Behavior

5) Research variation and heterogeneity: The results of the analysis show different values of variance and weights. The theory expressed by [20] is that the weight in a study is directly proportional to the number of research subjects (research samples). Research with 430 subjects will have a greater weight than research with 41 subjects. In addition to the number of subjects, the weight is also influenced by variations in the data. The weights are inversely proportional to the variation of the data. Research with more varied data will have a greater weight than research with smaller variations.

The test results show high heterogeneity, so that using random-effect models in measuring the combined effect of the data in the data input model in the Revman 5.4.1 application. The results obtained are POR $=3.95$ with a $90 \%$ confidence interval (1.81-8.61). The combined effect also resulted in a $Z$ value of 3.45 and a $p$-value of $<$ 0.0001 . Statistically, the combined effect is significant if the $p$-value $<0.05$, and this means that the null hypothesis is rejected; in other words, there is a relationship between information media and adolescent sexual behavior so that it can be concluded that adolescents who are exposed to media information tend to be at risk of 3.95 times doing adolescent sexual behavior.

Eight studies analyzed using Revman 5.4.1 show a very high level of heterogeneity bias based on the funnel plot. This explains that the analyzed studies have data variations that are not too wide, wherein the study shows that the highest effect size is 0.9486 , and the lowest is 0.087 .

6) Analysis of research results: A search for journals from various sources eventually resulted in 50 articles, then an assessment of the quality of the journals using inclusion criteria was made, and 13 research articles met the inclusion criteria. In the 13 articles, articles related to the relationship between attitudes and adolescent sexual behavior can be analyzed in the RevMan 5.4.1 application; there are eight articles.

Based on the test results through the Revman 5.4.1 application, the POR value $=3.95$ with a $90 \%$ confidence interval of 1.81-8.61. The combined effect also resulted in a $\mathrm{Z}$ value of 3.45 and a $p$-value of $<0.006$. Statistically, the combined effect was significant if the $p$-value $<0.05$. This means that it can be concluded that the null hypothesis is rejected; in other words, there is a relationship between information media and adolescent sexual behavior, so it can be concluded that adolescents who are exposed to information media tend to be 3.95 times at risk of pre-marital sexual behavior.

7) Comparison of meta-analysis results: In the study results, eight related articles stated a relationship between information media and adolescent sexual behavior indicated by a $p$-value $<0.05$, i.e., 0.006 . Research stated a relationship between 6 articles with a sample of 1289 and 2 articles that had no relationship with 107.

According to the researcher's assessment, information media has a risky impact on adolescents because teenagers' curiosity about something is very high, sources of information that are easy for them to reach are friends and media (VCD porn, internet access). Almost every teenager has a cellphone, laptop, and other electronic media, so it is very easy to access videos that are not following their age so that the more students access pornography on electronic media, print, etc., the more they know about risky sexual behavior and are willing to carry out risky 
sexual behavior or channel their desires.

\section{Meta-analysis of Factors Most Related to Adolescent Sexual Behavior}

Based on the results of the meta-analysis, it is known that the three variables in the study, namely knowledge, attitudes, and information media, both have a statistical relationship with adolescent sexual behavior. Based on the results of the meta-analysis, it can also be seen that the information media variable has a stronger relationship than the knowledge and attitude variables. This can be seen from the combined $z$-value; the $z$-value of the knowledge variable is 3.01. Attitude is 2.06 , and the information media variable is 3.45 . In addition, the POR value on the information media variable is also greater than knowledge and attitudes. The POR value on the information media variable is 3.95 , so it can be concluded that adolescents who are exposed to information media tend to be 3.95 times at risk of engaging in pre-marital sexual behavior.

\section{E. Policy Studies Related to Research Results}

Based on the meta-analysis results in this study, it was stated that attitude knowledge and information media had a relationship with adolescent sexual behavior. The information media factor has a greater effect than the knowledge and attitude factor.

The recommended form of health promotion efforts is strengthening adolescents' knowledge about reproductive health problems on sexual behavior. The targets in this activity are students and college students. This activity is carried out using approaches such as providing information and education, clinical medical services including supporting examinations and referrals, counseling, healthy life skills education (PKHS), training, educators, peers, and peer counselors. In addition to being educated from an early age and equipped with healthy life skills, education is skilled in developing their potential to live creatively and productively.

\section{CONCLUSION}

Based on the results of the meta-analysis and data synthesis from previous research articles that match the inclusion criteria, it can be concluded that:

1. In the adolescent knowledge variable, the results showed that there was a relationship between adolescent knowledge and sexual behavior, which had a moderate effect, where adolescents who had low knowledge increased the risk or tended to be 2.07 times to engage in sexual behavior compared to adolescents who had a high level of knowledge.
2. In the attitude variable, the results showed that there was a relationship between adolescent attitudes and sexual behavior that had a moderate effect, where adolescents who had adolescents who had negative attitudes tended to be 2.17 times more likely to engage in sexual behavior compared to adolescents who had positive attitudes about sexual behavior.

3. In the information media variable, the results show that there is a relationship between information media and sexual behavior that has a moderate effect, were adolescents who are exposed to information media tend to be at risk of 3.95 times doing pre-marital sexual behavior

4. Of the three variables studied, the information media variable has a stronger effect than the knowledge and attitude variables towards adolescent sexual behavior.

\section{REFERENCES}

[1] S. W. Sarwono, Theories of Social Psychology. Jakarta, Indonesia: CV. Raja-wali, 2004.

[2] R. Amrillah, "Differences in behavior towards premarital sexual relations in terms of family role," 2006. [Online]. Available: https://bit.ly/3xngnKI

[3] V. M. Masae, I. F. Manurung, D. S. Tira et al., "Relationship of knowledge, attitude, and access to social media with sexual behavior of adolescent girls," Public Health Media, vol. 1, no. 1, pp. 31-38, 2019.

[4] Z. Szakaly and K. Peto, "Health behaviour, behaviour change and personalised diet: The concept of lifelong health," Journal of Advances in Health and Medical Sciences, vol. 4, no. 2, pp. 43-52, 2018. doi: https://doi.org/10.20474/jahms4.2.2

[5] A. K. Sherlyanita and N. A. Rakhmawati, "The influence and activity patterns of internet and social media use on students SMPN 52 Surabaya," Journal of Information Systems Engineering and Business Intelligence, vol. 2, no. 1, pp. 17-22, 2016.

[6] B. S. Kalimantan. (2019) Study of early marriage in the perspective of law, sociology and health in South Kalimantan. Regional Research and Development Agency for South Kalimantan Province. [Online]. Available: https://bit.ly/3rKN5oa

[7] S. Susanti and I. Apriyanti, "Relationship of family quality, understanding of religious values and knowledge of premarital sex with adolescent perceptions of free sex behavior," in Procedure of National Seminars \& International, Semarang, Indonesia, 2016.

[8] P. H. Setyaningsih, "The relationship of knowledge level of reproductive health with adolescent sexual behavior in students at Smk Sasmita Jaya 1 Pamulang," Edu Dharma Journal: Jurnal Penelitian dan 
Pengabdian Masyarakat, vol. 5, no. 1, pp. 87-96, 2021.

[9] N. W. Sari, "Factors associated with sexual behavior in adolescents," Jurnal Human Care, vol. 5, no. 3, pp. 813-826, 2020.

[10] A. P. Mangkunegara, Any-jemen Human Resources Company. Bandung, Indonesia: PT Remaja Rosdakarya, 2005.

[11] M. Borenstein, L. V. Hedges, J. P. Higgins, and H. R. Rothstein, Introduction to meta-analysis. New York, NY: John Wiley \& Sons, 2021.

[12] Y. L. Tasidjawam, E. C. Grace, and A. Kormpis, "The relationship between knowledge and attitudes about reproductive health with premarital sexual behavior in students at SMP Negeri 3 Manado," Public Health, vol. 8, no. 6, pp. 528-535, 2019.

[13] N. Istiqomah and H. B. Notobroto, "The influence of knowledge, self-control on premarital sexual behavior among teenagers at SMK Surabaya," Jurnal Biometrika dan Kependudukan, vol. 5, no. 2, p. 125-134, 2016.

[14] F. H, L. Suwarni, and H. A. D., "Determinants of premarital sexual behavior in adolescents in Pontianak City," Jurnal Vokasi Kesehatan, vol. 5, no. 2, pp. 107-114, 2019.
[15] H. Astuti, "The relationship of knowledge and attitude toward sexual behavior," Journal of Midwiferia, vol. 3, no. 2, pp. 1-6, 2017.

[16] D. Afriani, S. Masyitah, and S. Agustina, "Determinants of risk sexual behavior in vocational school student," Jurnal Keperawatan'Aisyiyah, vol. 7, no. 2, pp. 51-60, 2020.

[17] Y. I. Saputri, "Factors associated with teenage premarital sex behavior," Journal of Public Health Sciences, vol. 5, no. 1, pp. 52-62, 2016.

[18] Z. Ulfah, D. Nugroho, and Y. Dharmawan, "The relationship of knowledge and attitude with reproductive health practices of students at SMK "X" Kebumen District I Quarter," Jurnal Kesehatan Masyarakat (Undip), vol. 4, no. 4, pp. 265-274, 2016.

[19] J. A. Sterne and M. Egger, "Funnel plots for detecting bias in meta-analysis: guidelines on choice of axis," Journal of Clinical Epidemiology, vol. 54, no. 10, pp. 1046-1055, 2001. doi: https://doi.org/ 10.1016/S0895-4356(01)00377-8

[20] M. S. Dahlan. (2012) Introduction to metaanalysis accompanied by application of metaanalysis using Excel program. [Online]. Available: https://bit.ly/3irWZIn 